\title{
Erratum to: Activation of liver $X$ receptor inhibits OCT2-mediated organic cation transport in renal proximal tubular cells
}

\author{
Teerasak Wongwan $^{1,2} \cdot$ Suticha Kittayaruksakul ${ }^{1} \cdot$ Nithi Asavapanumas $^{1}$ • \\ Varanuj Chatsudthipong ${ }^{1} \cdot$ Sunhapas Soodvilai ${ }^{1,2,3}$
}

Published online: 24 August 2017

(C) Springer-Verlag GmbH Germany 2017

Erratum to: Pflugers Arch - Eur J Physiol

DOI 10.1007/s00424-017-2033-8

The original publication of this article contained an error in

Fig. $6 \mathrm{~b}$. The correct version of Fig. $6 \mathrm{~b}$ can be seen below. The

original article has been corrected.

a
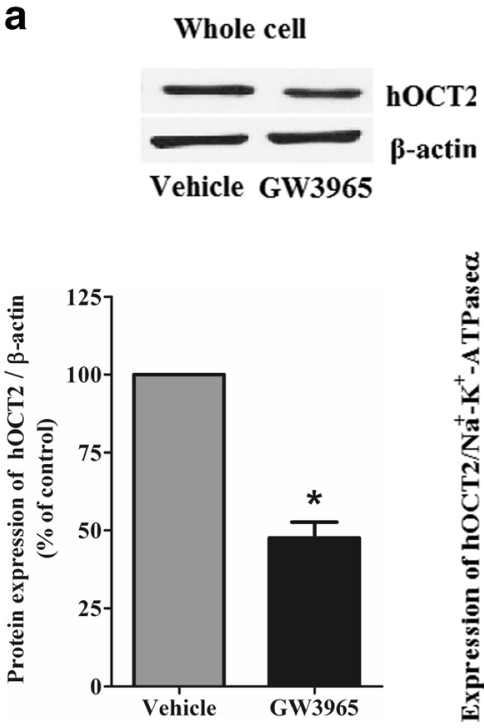

Membrane

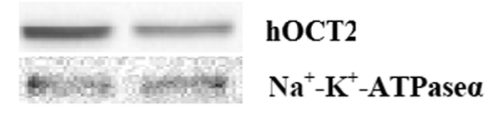

Vehicle GW3965

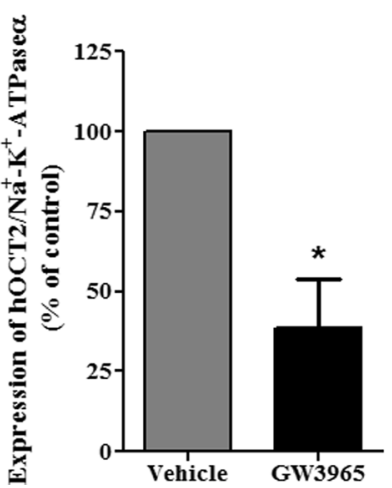

b

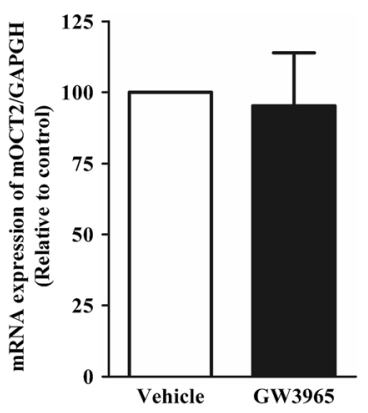

The online version of the original article can be found at http://dx.doi.org/ 10.1007/s00424-017-2033-8

Sunhapas Soodvilai

sunhapas.soo@mahidol.ac.th

1 Department of Physiology, Faculty of Science, Mahidol University, Rama VI Road, Rajathevi, Bangkok 10400, Thailand

2 Research Center of Transport Proteins for Medical Innovation, Faculty of Science, Mahidol University, Rajathevi, Bangkok 10400, Thailand

3 Excellent Center for Drug Discovery, Mahidol University, Rama VI Road, Rajathevi, Bangkok 10400, Thailand 